\title{
A TYCHONOFF NON-NORMAL SPACE
}

\author{
V. TZANNES \\ Department of Mathematics \\ University of Patras \\ Patras 26110, Greece \\ (Received July 13, 1992 and in revised form February 3, 1993)
}

\begin{abstract}
A Tychonoff non-normal space is constructed which can be used for the construction of a regular space on which every weakly continuous (hence every $\boldsymbol{\theta}$-continuous or $\eta$-continuous) map into a given space is constant.
\end{abstract}

KEY WORDS AND PHRASES. Tychonoff, non-normal, weakly, $\theta-, \eta$-continuous maps.

1980 AMS SUBJECT CLASSIFICATION CODE. 54D15, $54 \mathrm{C30}$.

\section{INTRODUCTION.}

We construct for every Hausdorf space $R$ a Tychonoff non-normal space $S$ such that if $f$ is a weakly continuous map of $S$ into $R$ then there exist two closed subsets $K^{\prime}, L^{\prime}, K^{\prime} \cap L^{\prime}=\emptyset$ such that $f\left(K^{\prime}\right)=f\left(L^{\prime}\right)=\{r\}, r \in R$. Therefore, applying the method of Jones [1], we (an first construct a regular space containing two points $-\infty$, $+\infty$ such that $f(-\infty)=f(+\infty)$, for every weakly continuous map $f$ of this space into $R$ and then, applying the method of Iliadis and Tzannes [2], a regular space on which every weakly continuous (hence every $\theta$-continuous or $\eta$-continuous (Dickman, Porter and Rubin (3])) map into $R$ is constant. The construction of $S$ is a modification of the space $T_{1}(R)$ in Iliadis and Tzannes [2]. For regular spaces on which every continuous map into a given space is constant see also Armentrout [4], Brandenburg and Mysior [5], van Douwen [6], Herrlich [7], Hewitt [8], Tzannes [9] and Jounglove [10]. A map $f: X \rightarrow Y$, where $X, Y$ are topological spaces is called 1) weakly continuous if for every $x \in X$ and $U$ open neighbourhood of $f(x)$ there exists an open neighbourhood $V$ of $x$, such that $f(V) \subseteq \mathrm{Cl} U, \quad$ 2) $\theta$-continuous if for every $x \in X$ and open neighbourhood $U$ of $f(x)$, there is an open neighbourhood $V$ of $x$ such that $f(\mathrm{Cl} V) \subseteq \mathrm{Cl} U \quad 3) \eta$-continuous if for every regular-open sets $U, V$ of $Y$,

$$
\text { (i) } f^{-1}(U) \subseteq \operatorname{IntCl} f^{-1}(U)
$$

(ii) $\operatorname{IntCl} f^{-1}(U \cap V) \subseteq \operatorname{IntCl} f^{-1}(U) \cap \operatorname{IntCl} f^{-1}(V)$.

Every $\eta$-continuous is $\theta$-continuous (Dickman, Porter and Rubin [3, Proposition 3.3. (c)]) and every $\theta$-continuous is obviously weakly continuous.

We denote 1$)$ by $|X|$ the cardinality, of $X, 2)$ by $\psi(X)=\sup \{\psi(X, x): x \in X\}$ the pseudocharacter of $X$, where $\psi(X, x)$ is the pseudocharacter of $X$ at $x$, that is the minimal cardinality of pseudobases of $x$. (The set $U_{\alpha}$ consisting of open neighbourhoods of $x$, is called a pseudobasis if $\left.\cap U_{\alpha}=\{x\}\right), 3$ ) by $\psi^{+}(X)$ the smallest cardinal number greater than $\psi(X)$.

2. THE SPACE $S$.

Let $R$ be a Hausdorff space and $K, L$ two uncountable sets such that $|K|=|L|=N>|R|$. 
For every $k_{i} \in K$ (resp. $l_{i} \in L$ ) we consider an uncountable set $K_{i}$ (resp. $L_{i}$ ) and a set $M$ such that $\left|K_{i}\right|=\left|L_{i}\right|=|M| \geq \psi^{+}(R)$. On the set $S=M \cup K U \cup K_{i} \cup L U \cup L_{i}$ we define the following topology: Every point belonging to $K_{i}, L_{i}$ is isolated. For every $k_{i} \in K$ (resp. $l_{i} \in L$ ) a basis of open neighbourhoods are the sets $O\left(k_{i}\right)=\left\{k_{i}\right\} \cup C_{i}$ (resp. $\left.O\left(l_{i}\right)=\left\{l_{i}\right\} \cup D_{i}\right)$, where $C_{i}, D_{i}$ consist of all but finite number of elements of $K_{i}, L_{i}$, respectively. For every point $m \in M$ a basis of open neighbourhoods are the sets $O(m)=\{m\} \cup P \cup Q$, where $P, Q$ contain all but finite number of elements of the sets $\left\{h_{i}(m): i \in I\right\},\left\{g_{i}(m): i \in I\right\}$, respectively, where $I$ is an index set, $|I|=N$ and $h_{i}, g_{i}$ are one-to-one maps of $M$ onto $K_{i}, L_{i}$, respectively.

One can show that the space $S$ is Tychonoff and non-normal.

Let $f$ be weakly continwous map of $S$ into $R$ Since $|K|>|R|$, it follows that for some $r_{1} \in R$ there exists $K^{\prime} \subseteq K$ such that $\left|K^{\prime}\right|=|K|$ and $f\left(K^{\prime}\right)=\left\{r_{1}\right\}$. Let $\left\{k_{n}: n=1,2, \ldots\right\}$ be a countable subset of $K^{\prime}$. Since for every open neighbourlood $U$ of $r_{1}$ the set $f^{-1}(C l U)$ contains an open neiglibourhood of $k_{n}, n=1,2, \ldots$, it follows that $\left|K_{n}\right| f^{-1}\left(r_{1}\right) \mid \leq \psi\left(R, r_{1}\right)$. Consequently, if $h_{n}$ is the one-to-one map of $M$ onto $K_{n}$ then $\left|h_{n}^{-1}\left(K_{n} \mid f^{-1}\left(r_{1}\right)\right)\right| \leq \psi\left(R, r_{1}\right)$ and lience $\left|\bigcup_{n=1}^{\infty} h_{n}^{-1}\left(K_{n} \mid f^{-1}\left(r_{1}\right)\right)\right| \leq \psi\left(R, r_{1}\right)$. Repeating all the above for the set $L$ we have that for some $r_{2} \in R$ there exist $L^{\prime} \subseteq L,\left|L^{\prime}\right|=|L|, f\left(L^{\prime}\right)=\left\{r_{2}\right\}$ and a countable subset $\left\{l_{n}: n=1,2, \ldots\right\} \subseteq$ $L_{-\infty}^{\prime}$ such that if $V$ is an open meighbourlood of $r_{2}$ then $\left|L_{n}\right| f^{-1}\left(r_{2}\right) \mid \leq \psi\left(R, r_{2}\right)$ and hence $\bigcup_{n=1}^{\infty} g_{n}^{-1}\left(L_{n} \backslash f^{-1}\left(r_{2}\right)\right) \mid \leq \psi\left(R, r_{2}\right)$. Therefore if $M^{\prime}=\bigcup_{n=1}^{\infty}\left(h_{n}^{-1}\left(K_{n} \backslash f^{-1}\left(r_{1}\right)\right) \cup g_{n}^{-1}\left(L_{n} \backslash f^{-1}\left(r_{2}\right)\right)\right)$ then $M \backslash M^{\prime} \neq 0$. Let $m \in M \backslash M^{\prime}$ and $C l W$ be a closed meighbourhood of $f(m)$ such that $r_{1}, r_{2} \notin \mathrm{ClW}$. There exists an open neighbourhood $O(m)$ of $m$ such that $f(O(m)) \subseteq \mathrm{ClW}$, while for every $n=1,2, \ldots, h_{n}(m) \in f^{-1}\left(r_{1}\right), g_{n}(m) \in f^{-1}\left(r_{2}\right)$ which imply that $f(m)=r_{1}=r_{2}$.

\section{REFEIRENCES}

1. JONES, F.B. Hereditarily separable, non-completely regular spaces, Proceedings of the Blacksburg Virginia Topological Conference, Sprimser-Verlag (375), 149-151.

2. ILIADIS, S. and TZANNES, V. Spaces on which every continuous map into a given space is constant, Can. J. Math. 38 (1986), 1281-1296.

3. DICKMAN, R.F. Jr., PORTER, J.R. and RUBIN, L.R. Completely regular absolutes and projective objects, Pacific J. Mothe 24 (2) (1981), 277-295.

4. ARMENTROUT, S. A Moore space on which every real-valued continuous function is comstant, Proc. Auner Math. Soc, 12 (1961), $106-109$.

5. BRANDENBURG, H. and MYSIOR, A. For every Hausdorff space $Y$ there exists a non - trivial Moore space on which all continuous functions into $\boldsymbol{Y}$ are constant, Pacific J.Math. 1 (1984), 1-8.

6. VAN DOUWEN, E.K. A regular space on which every continuous real-valued function is constant, Nieuw Archief roor Wiskunde 20 (1972), 143-145.

7. HERRLICH, H. Wann sind alle stetigen Abbildungen in $Y$ Konstant? Math. Zeitschr. 0 (1965), 152-154.

8. HEWITT, E. On two problems of Urysolin, Ammals of Matleenatics 47 (1946), 503-509.

9. TZANNES, V. A Moore strongly rigid space, Canl. Math. Bull. (34) (4) (1991), 547-552.

10. YOUNGLOVE, J.N. A locally connected, complete Moore space on which every real -valued continuous function is constant, Proc. Amer. Math. Soc. 20 (1969) 527-530 


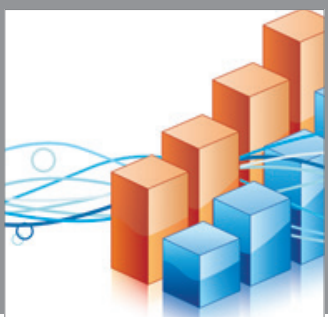

Advances in

Operations Research

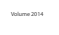

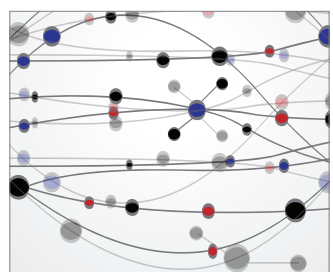

\section{The Scientific} World Journal
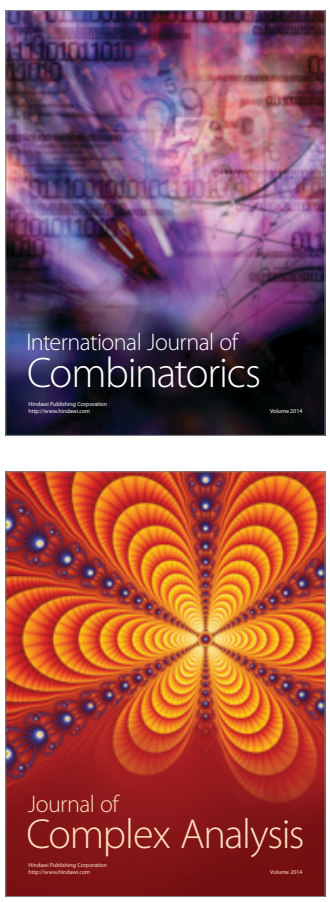

International Journal of

Mathematics and

Mathematical

Sciences
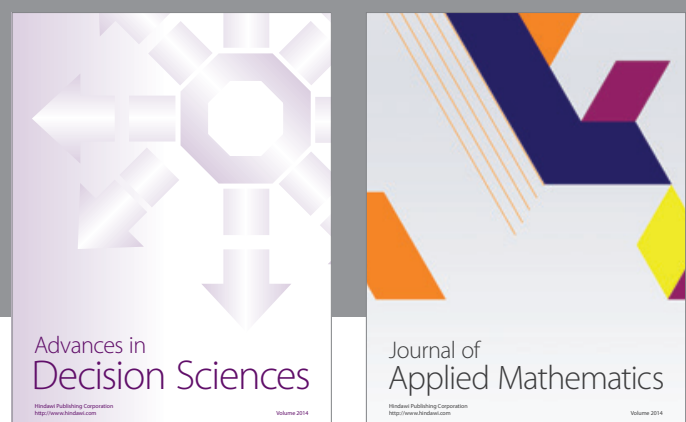

Journal of

Applied Mathematics
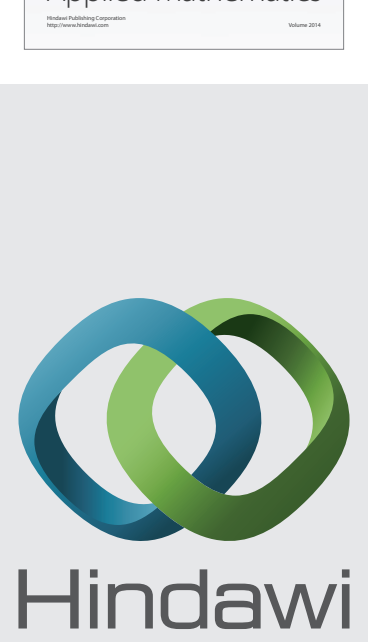

Submit your manuscripts at http://www.hindawi.com
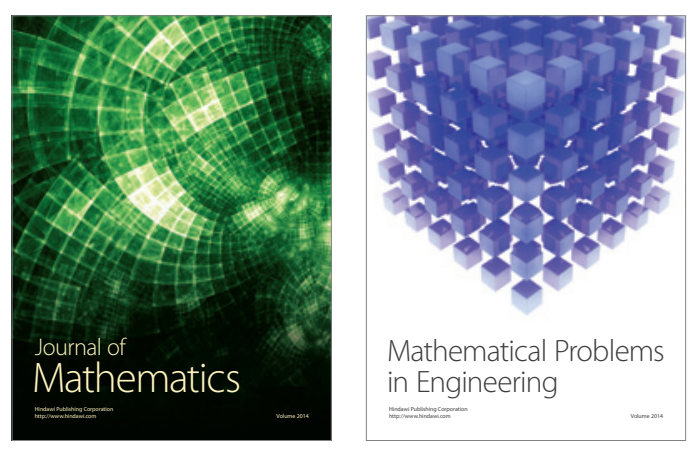

Mathematical Problems in Engineering
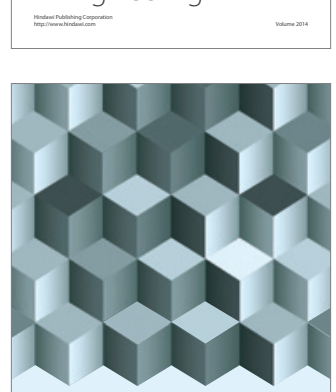

Journal of

Function Spaces
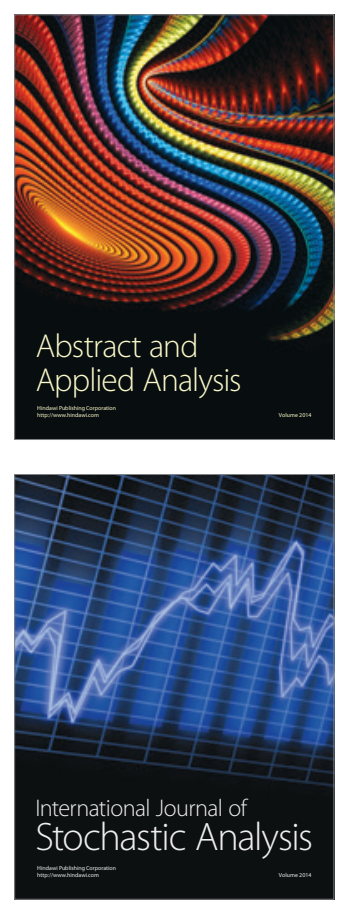

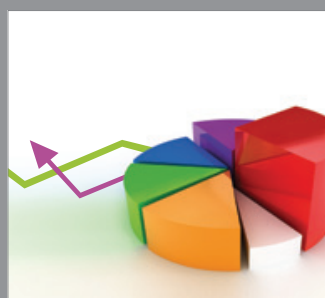

ournal of

Probability and Statistics

Promensencen
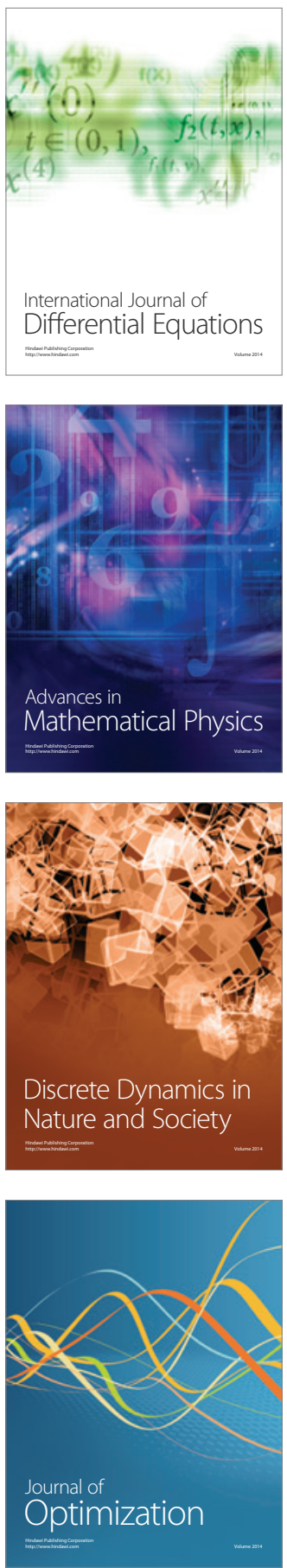